\title{
Tumoral masses of the ankle tendon sheath
}

\section{Luminița Varzaru}

Centre of Rheumatic Diseases “Doctor Ion Stoia”, Bucharest, Romania

Male patient, 55 years old, with no significant family or medical history, was admitted in our clinic for tenderness and swollen left ankle. The symptoms onset was two weeks prior admission, with no response at NSAID. The clinical examination showed no pathological findings, but pain and swelling on palpation, mild elevation of local temperature, with no change in color at left ankle. Laboratory tests revealed biological inflammatory syndrome (ESR $56 \mathrm{~mm} / \mathrm{h}$, CRP $62.22 \mathrm{mg} / \mathrm{l}$ ); the others test being in normal range (hemoglobin, leucocytes, thrombocytes, creatinine, blood glucose level, uric acid, rheumatoid factor, anti-CCP).

Ultrasound examination of the left ankle showed pathological findings at the posterior tibial tendon sheath (fig1): two tumoral masses, one of $1.38 / 0.36 \mathrm{~cm}$ and the other one of $0.29 / 0.15 \mathrm{~cm}$ in size, with the same characteristics- well defined, hyperechoic, discrete heterogeneous, with acoustic shadowing, without Doppler signal. In addition, an anechoic, homogeneous halo along the tendon course, displaceable under transducer, without Doppler signal was found. There were no other significant changes at ultrasonography.

\section{Questions:}

1. What is the diagnosis?

2. What other investigations do you consider necessary for the final diagnosis?

3. What are the particular features of the case?
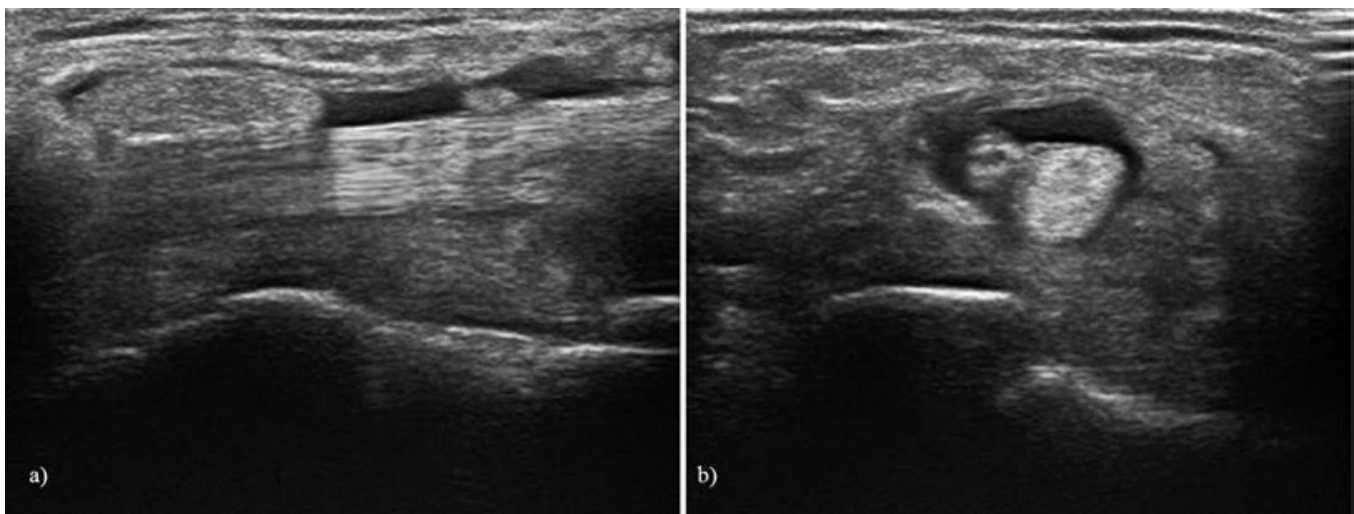

Fig 1. Gray scale a) longitudinal scan and b) transversal scan of the posterior tibial tendon (retromaleolar, medial compartment of left ankle)

Received Accepted

Med Ultrason

2013, Vol. 15, No 2, 164-165

Corresponding author: Luminița Varzaru

5 Thomas Masaryk Street, 020983

Bucharest, Romania

Phone: +40212116848

Email: luminita_v2000@yahoo.com 


\section{Answer QUIZ vol 15, no. 1}

\section{Boy with renal failure after renal transplant}

\section{Aurel Bizo}

II ${ }^{\text {rd }}$ Pediatric Department, Cluj-Napoca, Romania sented by the results of the urine cultures which revealed a massive infestation with Candida albicans.

Final diagnosis: Renal graft micosis (candidiasis)

The ultrasonographic diagnosis is confirmed by the aspect of the explantation specimen which showed shiny candida hyphae after it was sliced.

\section{Differential diagnosis:}

Renal lithiasis - very unlikely

Anaerobic pyelonephritis - the highly dramatic presentation was not suggesting this diagnosis

Malakoplakia - the age of the patient and the presen-

tation are uncharacteristic

\section{Establish the diagnosis in this case}

An element suggestive for the diagnosis was repre- 